PROCEEDINGS OF THE

AMERICAN MATHEMATICAL SOCIETY

Volume 129, Number 9, Pages 2799-2802

S 0002-9939(01)05854-3

Article electronically published on February 9, 2001

\title{
THE KANENOBU-MIYAZAWA CONJECTURE AND THE VASSILIEV-GUSAROV SKEIN MODULES BASED ON MIXED CROSSINGS
}

\author{
JÓZEF H. PRZYTYCKI AND KOUKI TANIYAMA \\ (Communicated by Ronald Fintushel) \\ In memory of Mikhail Gusarov (August 3, 1958 - June 25, 1999)
}

\begin{abstract}
We show that a Brunnian link of $n$ components and the $n$ component trivial link share the same first $n-1$ coefficients of the Jones-Conway (Homflypt) polynomial (answering the question of Kanenobu and Miyazawa). We prove also the similar result for the Kauffman polynomial of Brunnian links. We place our solution in the context of Vassiliev-Gusarov skein modules based on mixed singular crossings.
\end{abstract}

\section{Kanenobu-Miyazawa conjecture}

A Brunnian link of $n$ components is an oriented link such that the removal of any of its components leaves us with a trivial link of $n-1$ components (for $n=2$ we assume also that the linking number is equal to 0 ).

Let $P_{L}(v, z) \in Z\left[v^{ \pm 1}, z^{ \pm 1}\right]$ be the Jones-Conway (Homflypt) polynomial of oriented links in $S^{3}$. That is, $P_{T_{n}}(v, z)=\left(\frac{v^{-1}-v}{z}\right)^{n-1}$ for a trivial link of $n$ components, $T_{n}$, and $v^{-1} P_{L_{+}}-v P_{L_{-}}=z P_{L_{0}}$ for a Conway skein triple $L_{+}, L_{-}$and $L_{0}$.

Let $\tilde{P}_{L}(v, z)=z^{n-1} P_{L}(v, z)$ for a link $L$ of $n$ components. $\tilde{P}_{L}(v, z)$ satisfies: $\tilde{P}_{T_{n}}(v, z)=\left(v^{-1}-v\right)^{n-1}$ and

$$
v^{-1} \tilde{P}_{L_{+}}-v \tilde{P}_{L_{-}}= \begin{cases}z^{2} \tilde{P}_{L_{0}} & \text { for a mixed crossing } \\ \tilde{P}_{L_{0}} & \text { for a self-crossing. }\end{cases}
$$

In particular $\tilde{P}_{L}(v, z) \in Z\left[v^{ \pm 1}, z\right]$ and $z$ is not an invertible element in the ring. Theorem 1.1 (Kanenobu-Miyazawa conjecture). If $L$ is a Brunnian link of $n$ components, then

$$
\tilde{P}_{L}-\tilde{P}_{T_{n}} \equiv 0 \bmod \left(z^{2 n-2}\right) .
$$

Proof. Consider a diagram $D_{L}$ of a nontrivial Brunnian link $L$ of $n$ components ( $n \geq$ 2 ) which consists of a trivial link $T_{n-1}$ (no crossings) with components $L_{1}, \ldots, L_{n-1}$, and a component $L_{n}$ which intersects all other components. Let $S_{i}$ denote the subset of crossings of $L_{i}$ and $L_{n}$ at which $L_{i}$ is below $L_{n}$. We use the notation $L_{\epsilon_{1}, \ldots, \epsilon_{n-1}}^{S_{1}, \ldots, S_{n-1}}$ where $\epsilon_{i}=-1$ or 1 , to denote a link obtained from $L$ by changing all

Received by the editors August 24, 1999 and, in revised form, January 14, 2000.

2000 Mathematics Subject Classification. Primary 57M27.

The first author was partially supported by NSF grant DMS-9808955. 
the crossings in $S_{i}$ of the diagram $D_{L}$ iff $\epsilon_{i}=-1$. In particular $L_{1, \ldots, S_{n-1}}^{S_{1}, \ldots}=L$. On the other hand if not all $\epsilon_{i}=1$, then $L_{\epsilon_{1}, \ldots, \epsilon_{n-1}}^{S_{1}, \ldots, S_{n-1}}$ is a trivial link of $n$ components, because when $\epsilon_{i}=-1$, then $L_{i}$ is above the rest of the link. Therefore

$$
\tilde{P}_{L}-\tilde{P}_{T_{n}}=\sum_{\epsilon_{1}, \ldots, \epsilon_{n-1}} \epsilon_{1} \cdots \epsilon_{n-1} \tilde{P}_{L_{\epsilon_{1}, \ldots, \epsilon_{n-1}}^{S_{1}, \ldots, S_{n-1}}} .
$$

Now, because $l k\left(L_{i}, L_{j}\right)=0$ for Brunnian links, we can write the right-hand sum of the equation (using skein relation between mixed crossings) as:

$$
z^{2 n-2} \sum_{\epsilon\left(S_{1}\right), \ldots, \epsilon\left(S_{n-1}\right)}(-1)^{*} v^{*} \tilde{P}_{L_{\epsilon\left(S_{1}\right), \ldots, \epsilon\left(S_{n-1}\right)}^{S_{1}, \ldots, S_{n-1}}} .
$$

We should explain the notation and the formula will be clear (and our proof of Theorem 1.1 completed). We order crossings of $S_{i}$, say $q_{1}, q_{2}, \ldots, q_{k_{i}}$, and $\epsilon\left(S_{i}\right)$ denote the number between 1 and $k_{i}$ (i.e. $1 \leq \epsilon\left(S_{i}\right) \leq k_{i}$ ). The meaning of $L_{\epsilon\left(S_{1}\right), \ldots, \epsilon\left(S_{n-1}\right)}^{S_{1}, \ldots, S_{n-1}}$ is that we change crossings $q_{j}$ in $S_{i}$ if $j<\epsilon\left(S_{i}\right)$, we smooth the crossing $q_{\epsilon\left(S_{i}\right)}$ and we do not change other crossings of $S_{i}$. The exponents of -1 and $v$ depend on sign of changed/smoothed crossings but are inessential for us. The important thing to observe is that we always operate on mixed crossings, and every link of the sum is a knot obtained by $n$ smoothings of mixed crossings (and some changings) so finally we have the common power $z^{2 n-2}$.

We have the similar result for the Kauffman polynomial. We delay a proof of it because the result follows as a corollary of a more general theory.

Theorem 1.2. If $L$ is a Brunnian link of $n$ components, then

$$
\tilde{F}_{L}-\tilde{F}_{T_{n}} \equiv 0 \bmod \left(z^{2 n-2}\right)
$$

where $\tilde{F}_{L}=z^{n-1} F_{L}$ and $F_{L}$ is the Dubrovnik version of the Kauffman polynomial. That is, it satisfies: $F_{T_{k}}=\left(\frac{a-a^{-1}+z}{z}\right)^{k-1}$ and $a^{w\left(L_{+}\right)} F_{L_{+}}-a^{w\left(L_{-}\right)} F_{L_{-}}=$ $z\left(a^{w\left(L_{0}\right)} F_{L_{0}}-a^{w\left(L_{\infty}\right)} F_{L_{\infty}}\right)$ where $w(L)$ for a link diagram $L$ is the writhe (or Tait) number of $L$, that is, the sum of signs of all crossings of $L$.

\section{VASSILIEV-GUSAROV SKEIN MODULES BASED ON MIXED CROSSINGS}

Our proof is motivated by the notion of $n$-triviality of links, as introduced by Yamamoto [Y], Ohyama [O] and Gusarov [G]. This notion is related to VassilievGusarov skein modules (and the dual space of Vassiliev invariants), and JonesConway and Kauffman polynomials. The difference with respect to the standard treatment (see $[\overline{\mathrm{P}}]$ ) is that we consider only mixed singular crossings. We can develop our theory in any 3-manifolds, but we will limit ourselves here to $S^{3}$.

(1) Skein modules. Let $\mathcal{L}$ be the set of oriented links up to ambient isotopy, $R$ a commutatative ring with identity, and $R \mathcal{L}$ the free module over $\mathcal{L}$. Let $\mathcal{L}^{s g m}$ be the set of "mixed" singular links, that is, a set of singular links such that, for any $k$, smoothing $k$ singular crossings reduces the number of components by $k$. If we divide $R \mathcal{L}^{\text {sgm }}$ by the singular crossing splitting relations $\left(L_{s g m}=L_{+}-L_{-}\right)$, we get $R \mathcal{L}$ back. Let $C_{i}^{\text {mix }}$ be a submodule of $R \mathcal{L}$ generated by links with $i$ "mixed" singular crossings. We define the "mixed" Vassiliev-Gusarov skein module as the quotient $W_{k}^{\text {mix }}=R \mathcal{L} / C_{k+1}^{\text {mix }}$. 
(2) "Mixed" $n$-trivial links 11 A link $L$ of $m$ components $(m \geq n+1)$ is called a "mixed" $n$-trivial link if there is a diagram of $L$ with $n$ groups of crossings $S_{1}, \ldots, S_{n}$ such that $L_{\epsilon_{1}, \ldots, \epsilon_{n}}^{S_{1}, \ldots, S_{n}}$ is a trivial link of $m$ components with the possible exception of $L=L_{1}^{S_{1}, \ldots, S_{n}}$. We require furthermore the "mixed" crossing condition, that is, if $p_{i} \in S_{i}$ then the smoothing of the crossings $p_{1}, \ldots, p_{n}$ reduces the number of components of $L$ by $n$. It follows from the considerations in the first section that:

Lemma 2.1. A Brunnian link of $n$ components is "mixed" $n-1$ trivial.

\section{(3) "Mixed" $n$-trivial links and "mixed" skein modules.}

Theorem 2.2. If $L$ is a "mixed" $n$-trivial link of $m$ components $(m \geq n+1)$, then

$$
L-T_{m} \in C_{n}^{m i x}
$$

or equivalently $L=T_{m}$ in the "mixed" Vassiliev-Gusarov skein module $W_{n}^{\operatorname{mix}}$.

Proof. Following our proof of Theorem 1.1, but using singular links instead of smoothings, we can write (in $R \mathcal{L}$ ):

$$
\begin{aligned}
L-T_{m} & =\sum_{\epsilon_{1}, \ldots, \epsilon_{n}} L_{\epsilon_{1}, \ldots, \epsilon_{n}}^{S_{1}, \ldots, S_{n}} \\
& =\sum_{\delta\left(S_{1}\right), \ldots, \delta\left(S_{n}\right)} L_{\delta\left(S_{1}\right), \ldots, \delta\left(S_{n}\right)}^{S_{1}, \ldots, S_{n}} .
\end{aligned}
$$

Below we explain our notation.

We order the crossings of $S_{i}$, say $q_{1}, q_{2}, \ldots . q_{k_{i}}$, and $\delta\left(S_{i}\right)$ denote a number between 1 and $k_{i}$ (i.e. $1 \leq \delta\left(S_{i}\right) \leq k_{i}$ ). The meaning of $L_{\delta\left(S_{1}\right), \ldots, \delta\left(S_{n}\right)}^{S_{1}, \ldots, S_{n-1}}$ is that we change the crossings $q_{j}$ in $S_{i}$ if $j<\delta\left(S_{i}\right)$, we put a singular crossing in place of the crossing $q_{\delta\left(S_{i}\right)}$ and we do not change other crossings of $S_{i}$. Theorem 2.2 follows from the formula.

(4) Jones-Conway and Kauffman polynomials for "mixed" $n$-trivial links. Applying Theorem 2.2 we obtain:

Theorem 2.3. Let $L$ be a "mixed" $n$-trivial link of $m$ components. For $n=1$ assume additionally that $l k(L)=0$. Then:

(P) $\tilde{P}_{L}-\tilde{P}_{T_{m}} \equiv 0 \bmod \left(z^{2 n}\right)$.

(F) $\tilde{F}_{L}-\tilde{F}_{T_{m}} \equiv 0 \bmod \left(z^{2 n}\right)$ where $\tilde{F}_{L}=z^{m-1} F_{L}$ and $F_{L}$ is the Dubrovnik version of the Kauffman polynomial.

As a corollary we obtain the following generalization of the Kanenobu- Miyazawa conjecture.

After [S] we define an $n$-color Brunnian link $L$ as a link whose components are divided into $n$ nonempty groups $L_{1}, L_{2}, \ldots, L_{n}$ in such a way that $L-L_{i}$ is a trivial link and for $n=2, l k(L)=0$.

Corollary 2.4. Let $L$ be an $n$-color Brunnian link of $m$ components. Then

(T) $L$ is a "mixed" $n-1$ trivial link.

(P) $\tilde{P}_{L}-\tilde{P}_{T_{m}} \equiv 0 \bmod \left(z^{2 n-2}\right)$.

(F) $\tilde{F}_{L}-\tilde{F}_{T_{m}} \equiv 0 \bmod \left(z^{2 n-2}\right)$.

\footnotetext{
${ }^{1} \mathrm{In}$ choice of " $n$ " we follow $[\mathrm{O}$. In $[\mathrm{G}]$ or $[\underline{\mathrm{P}}$ it would be " $n-1$ ".
} 
(5) "Mixed" $n$-similarity. The notion of an $n$-trivial link is generalized in $\mathrm{T}, \mathrm{G}$, to the concept of an $n$-similar links. We adjust the concept to the "mixed" crossings case.

Definition 2.5. A link $L$ is "mixed" $n$-similar to a link $L_{1}$ if there is a diagram of $L$ with $n$ groups of crossings $S_{1}, \ldots, S_{n}$ such that $L_{\epsilon_{1}, \ldots, \epsilon_{n}}^{S_{1}, \ldots, S_{n}}$ represents the link $L_{1}$ with the possible exception of $L=L_{1, \ldots, 1}^{S_{1}, \ldots, S_{n}}$.

Theorems 2.2 and 2.3 generalize to the case of a "mixed" $n$-similarity.

Theorem 2.6. Let $L$ be a link "mixed" $n$-similar to $L_{1}$. Then:

(T) $L-L_{1} \in C_{n}^{\text {mix }}$.

Let additionally $l k(L)=l k\left(L_{1}\right)$ for $n=1$. Then:

(P) $\tilde{P}_{L}-\tilde{P}_{L_{1}} \equiv 0 \bmod \left(z^{2 n}\right)$.

(F) $\tilde{F}_{L}-\tilde{F}_{L_{1}} \equiv 0 \bmod \left(z^{2 n}\right)$.

"Mixed" $n$-similar links can be constructed from Brunnian links:

Example 2.7. Let $L$ be a Brunnian link of $n$ components $K_{1}, \ldots, K_{n}$ and $B_{1}, \ldots, B_{n}$ be links in a solid torus. Then the link $L_{1}$ obtained from $L$ by decorating every $K_{i}$ by $B_{i}$ is "mixed" $(n-1)$-similar to the link $L_{2}$ obtained by decorating a trivial link of $n$ components by $K_{1}, \ldots, K_{n}$ (informally $L_{2}$ is a disjoint sum of $K_{i}$ 's).

\section{REFERENCES}

[G] M.N.Gusarov, On $n$-equivalence of knots and invariants of finite degree, Topology of Manifolds and Varieties, 173-192, Advances in Soviet Mathematics 18, AMS, 1994. MR 96i:57005

[K-M] T.Kanenobu, Y.Miyazawa, The second and third terms of the HOMFLY polynomial of a link, Kobe J. Math., 16, 1999, 147-159. CMP 2000:09

[O] Y.Ohyama, A new numerical invariant of knots induced from their regular diagrams, Topology Appl., 37, 1990, 249-255. MR 92a:57009

[P] J.H.Przytycki, Vassiliev-Gusarov skein modules of 3-manifolds and criteria for periodicity of knots, Low-Dimensional Topology, Knoxville, 1992 ed.: Klaus Johannson International Press Co., Cambridge, MA 02238, 1994, 143-162. MR 96a:57026

[S] T.Stanford, Four observations on $n$-triviality and Brunnian links, preprint 1998 Journal of Knot Theory and Its Ramifications, 9, 2000, 213-219. CMP 2000:11

[T] K.Taniyama, On similarity of links, Gakujutsu Kenkyu, School of Education, Waseda University, Series of Mathematics, 41, 1993, 33-36.

[Y] M.Yamamoto, Knots in spatial embeddings of the complete graph on four vertices, Topology Appl., 36, 1990, 291-298. MR 91m:57007

Department of Mathematics, University of Maryland, College Park, Maryland 20742

(On leave from) Department of Mathematics, George Washington University, WashINGTON, DC 20052

E-mail address: przytyck@gwu.edu

Department of Mathematics, Tokyo Woman's Christian University, Zempukuji, SugiNAMIKU, TOKYO, 167-8585, JAPAN

E-mail address: taniyama@twcu.ac.jp 